\title{
Sistema experto de apoyo para el diagnóstico y tratamiento de la neumonía en cerdos
}

\author{
Expert support system for the diagnosis and treatment of pneumonia in pigs
}

\author{
Allan Steven Veloza Rodríguez \\ Facultad de Ingeniería, Universidad de Cundinamarca, Grupo de investigación de sistemas y tecnología Facatativá \\ GITSFA, Cundinamarca, Colombia. \\ allanveloza88@gmail.com
}

\begin{abstract}
Resumen- Por medio de un sistema experto es posible diagnosticar rápida y eficazmente según los síntomas que tenga el cerdo, el nivel de gravedad de la neumonía, esto con ayuda del conocimiento que provea el veterinario encargado en esta área y desarrollando así un sistema experto que ayude a la toma de decisiones para tratar a tiempo esta enfermedad tan común que sufre la especie porcina. Para el desarrollo de la aplicación es necesario tener una serie de reglas o motor de inferencia que es el que permite la toma de decisiones del sistema.
\end{abstract}

Los aplicativos web han tenido gran impacto en la sociedad porque con ellos se tiene a la mano todo tipo de información; al tener un sistema experto almacenado allí permitirá al usuario acceder a una herramienta experta, quien lo apoyara en un correcto diagnóstico $y$ tratamiento para la enfermedad; minimizando perdidas en la producción.

Palabras claves- Sistema experto, neumonía, motor de inferencia, base de conocimiento.

\begin{abstract}
Through an expert system, it is possible to diagnose in a fast and accurate manner symptoms of pneumonia that a pig can have, as well as the level of seriousness of the illness. This can be achieved with the aid and knowledge of a veterinarian in charge of this area. Developing an expert system to aid the decision making could treat this illness that is very common among pigs. In order to develop this application it is necessary to have certain rules or an inference engine, which allows the expert system to make decisions.
\end{abstract}

The web applications have had a great impact on society. Having an expert system is like having a tool with a lot of information stored, which in the end can help the user give an accurate diagnosis and treatment of the illness, minimizing production losses.

Key Word- Expert system, pneumonia, inference engine, base of knowledge.

\section{INTRODUCCIÓN}

La llegada de la tecnología a las fincas productoras de cerdos, puede constituirse como un gran apoyo y ventaja en el momento de detectar la neumonía en cerdos a tiempo; que un sistema experto suministre al usuario un diagnóstico y tratamiento a tiempo con el fin de no tener pérdidas en la producción de este tipo de carne. No obstante, se debe tener en cuenta que no hay acceso a un veterinario todos los días quien diagnosticaría y trataría la neumonía que pone en peligro la salud del animal. [1].

Las fincas productoras se encuentran situadas en áreas rurales lo que dificulta el acceso del veterinario todo el tiempo sin embargo están los trabajadores quienes podrían apoyarse en el sistema para poder aplicar un tratamiento que impida la evolución de enfermedades graves en el animal.

Por otra parte, al ser un aplicativo web, este permite tener un acceso rápido y eficaz al sistema de una manera sencilla para el usuario, teniendo en cuenta que este tipo de sistema es nuevo para este entorno puesto que no se cuenta con ningún tipo de apoyo sistematizado. [2]

\section{DESARROLLO DEL TEMA}

Para lograr una buena producción de animales para el consumo humano es vital que este tipo de granjas productoras se encuentren en lugares retirados, ya que se generan malos olores y podría afectar la salud de las personas; al estar ubicado en zonas rurales en ocasiones se dificulta el acceso de un veterinario que dé una pronta solución si algún animal enferma, es decir no son atendidos a tiempo corren el riesgo de ponerse graves y perder peso o incluso morir. [3] 
La modernización de la ganadería tiene que ser parte de la modernización del sector agropecuario en general, ya que los componentes de dichos sectores están estrechamente relacionados. La ganadería no existe como una actividad independiente de otras formas de uso de la tierra. A su vez, la modernización del sector agrícola se concibe como la generación de sistemas de uso de la tierra productivos, rentables, ecológicamente sostenibles, e insertos en cadenas de producción, servicios y comercialización eficientes. Esta sólo será posible dentro de un proceso de modernización de la sociedad en general. [4]

\section{LA NEUMONIA}

El índice de enfermedades porcinas varia de granja a granja, sin embargo, se han realizado estudios a diferentes granjas que estadísticamente muestran que la amenaza sanitaria más frecuente fue el PRRS (Virus del Síndrome Respiratorio y Reproductivo Porcino), También fue votado como la principal causante de pérdidas La influenza porcina también fue de las más votadas por eso la inclinación por esta enfermedad ya que es de las más frecuentes para e desarrollo del sistema experto. [12]

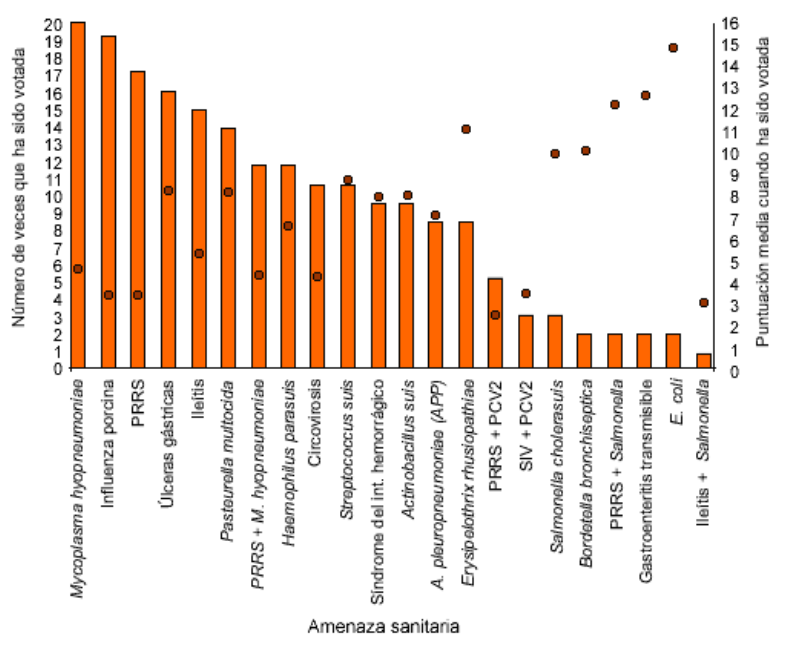

Grafica 1. Comparación Enfermedades Porcinas

\section{TIPOS DE NEÚMONIA PORCINA}

NEUMONÍA ENZOÓTICA: La neumonía enzoótica, se produce en todo el mundo y ha sido conocido desde hace muchos años. El organismo, con sus características típicas, invade el tracto respiratorio en varias etapas sucesivas. La relevancia económica de la neumonía está influenciada en gran medida por las infecciones secundarias. Diferentes pruebas para el diagnóstico de neumonía en cerdos individuales y en grupos están disponibles. El tratamiento y el control no es sencilla ya que la neumonía enzoótica es una enfermedad multifactorial. [5]

PLEURONEUMONÍA PORCINA: Enfermedad de mayor impacto económico la cual presenta varios factores de virulencia que las hacen difícil de controlar o eliminar por los mecanismos inmunológicos normales. Es de elevada diseminación, altamente contagiosa y en muchas ocasiones letal en cerdos desde el destete al sacrificio. Provoca en el 30$50 \%$ de los cerdos una pleuritis fibrinosa con adherencias costales bastante características y elevada mortalidad en cuadros agudos, con importante retraso del crecimiento en las formas crónicas. [6]

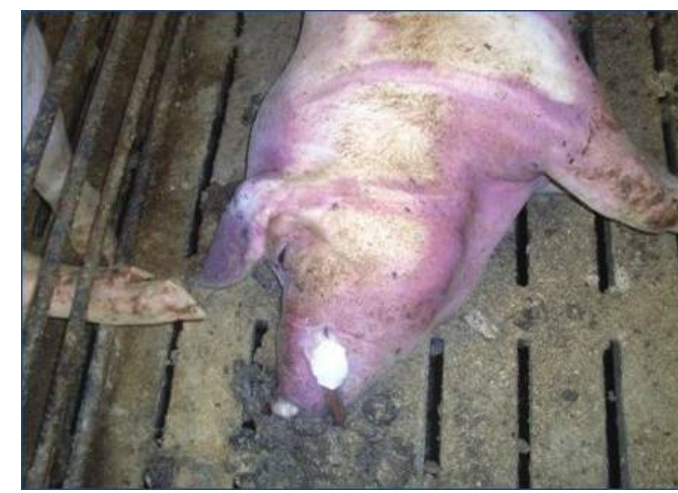

Figura 1. Cerdo infectado de pleuroneumonía porcina Tomado de:

http://www.aacporcinos.com.ar/articulos/sanidad_porcina_08012_actinobacillus_pleuropneumoniae_en_porcino.html

NEUMONÍA POR SALMONELLA: Causada por Salmonella la que por vía sanguínea (septicemia) invaden los pulmones; la cepa más aislada de pulmones afectados es S. cholerasuis variedad Kunzandor. Generalmente se presenta en cerdos después del destete y menores de cinco meses. Morbilidad menor del $10 \%$ y alta mortalidad en cerdos no tratados. Los cerdos afectados presentan además la clínica y lesiones descrita en la salmonelosis septicémica. [7]. 


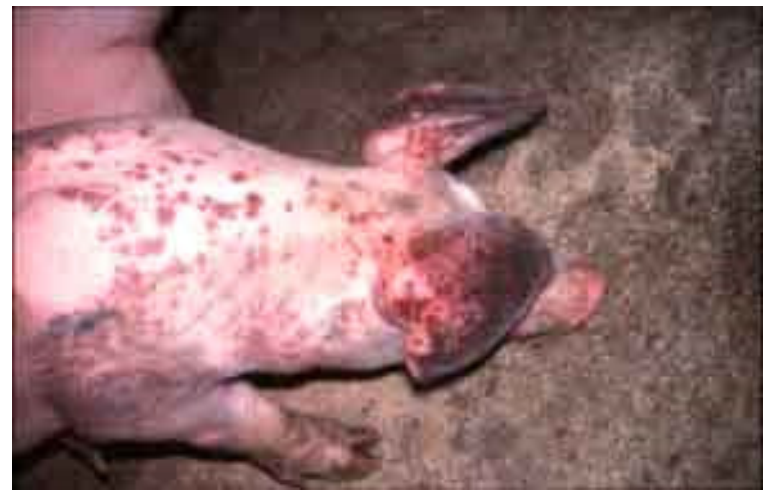

Figura 2. Cerdo infectado por salmonella Tomado de: https://www.engormix.com/MA-

porcicultura/sanidad/articulos/que-sabemos-acerca-circovirus-t213/165p0.htm

NEUMONÍA VERMINOSA PORCINA: Por la presencia y acción de larvas migratorias o por la presencia y acción de bacterias en los bronquios pulmonares) los que crean las condiciones favorables para la colonización de otros agentes perjudiciales para la salud del animal. [8]

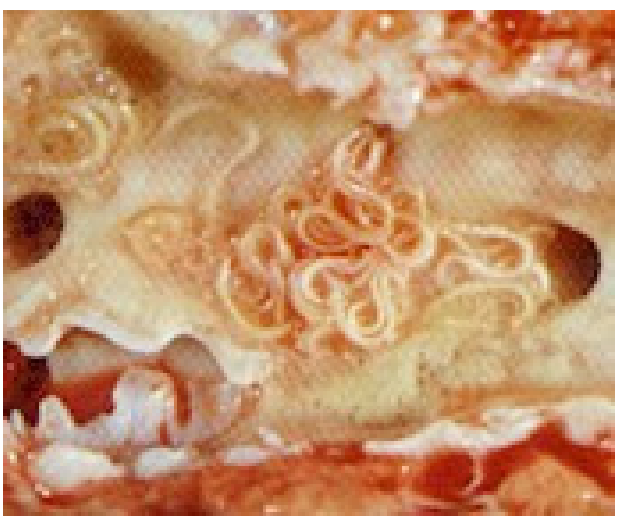

Figura 3. Pulmon abierto infectado con larvas

Tomado de:

http://parasitipedia.net/index.php?option=com_content $\&$ view=article $\&$ id $=16$ 9\&Itemid $=248$

\section{SISTEMA EXPERTO}

Teniendo en cuenta la importancia de la producción animal para el consumo humano y el nivel de calidad que este debe tener se hace necesario un sistema experto que facilite el diagnóstico y tratamiento de enfermedades en la especie porcina para que no haya pérdida en la producción, además es de vital importancia que los animales estén completamente sanos a la hora de consumirlos.

En la universidad privada Islamic Azad University de Iran, en el departamento de inteligencia artificial, viendo la importancia que representa la salud humana y como está ligada a la salud de los animales domésticos, en el artículo publicado en la revista IEEE "A FUZZY EXPERT SYSTEM TO DIAGNOSE DISEASES WITH NEUROLOGICAL SIGNS

IN DOMESTIC ANIMAL" destacan el uso de lógica difusa para el diagnóstico de enfermedades neurológicas en animales domésticos, ya que la detección sintomatológica de estas es compleja y el veterinario suele dudar de su dictamen, asi con la ayuda de lógica difusa lograr separar o incluir síntomas que sean realmente de enfermedades neurológicas. [9]

En la institución pública Universidad de Córdova ubicada en el caribe colombiano podemos encontrar un sistema experto que puede diagnosticar plagas y enfermedades en cultivos de berenjena, denotando así mismo el compromiso que tiene esta universidad con el desarrollo agropecuario colombiano, el artículo se llama "SISTEMA EXPERTO PARA EL DIAGNOSTICO DE PLAGAS Y ENFERMEDADES EN LOS CULTIVOS DE BERENJENA (Solanum Melongena L.) EN LA REGIÓN CARIBE DE COLOMBIA", de la revista Ingeniería e Innovación. Se destaca el uso de herramientas de fuzuficacion como el Prolog, y desarrollo como Java. [10]

\section{COMPARACIÓN}

A continuación, se presenta una comparación entre los 4 tipos de neumonía y sus diferentes síntomas.

\begin{tabular}{|c|c|c|c|c|}
\hline & $\begin{array}{c}\text { NEUMONÍA } \\
\text { ENZOÓTICA } \\
\text { PORCINA }\end{array}$ & $\begin{array}{c}\text { PLEURO } \\
\text { NEUMONÍA } \\
\text { PORCINA }\end{array}$ & $\begin{array}{c}\text { NEUMONÍA } \\
\text { POR } \\
\text { SALMONELLA }\end{array}$ & $\begin{array}{l}\text { NEUMONÍA } \\
\text { VERMINOSA } \\
\text { PORCINA }\end{array}$ \\
\hline $\begin{array}{l}\text { Falta de } \\
\text { apetito } \\
\text { (anorexia) }\end{array}$ & $\mathbf{X}$ & $\mathbf{X}$ & & \\
\hline Fiebre. & $\mathbf{X}$ & $\mathbf{X}$ & $\mathbf{X}$ & $\mathbf{X}$ \\
\hline 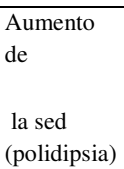 & & & $\mathbf{X}$ & $\mathbf{X}$ \\
\hline Postración & $\mathbf{X}$ & $\mathbf{X}$ & & \\
\hline $\begin{array}{l}\begin{array}{l}\text { Aumento } \\
\text { de }\end{array} \\
\text { la } \\
\text { frecuencia } \\
\text { respiratoria } \\
\text { (disnea) }\end{array}$ & $\mathbf{X}$ & $\mathbf{X}$ & $\mathbf{X}$ & \\
\hline Estornudos & & $\mathbf{X}$ & & $\mathbf{X}$ \\
\hline
\end{tabular}




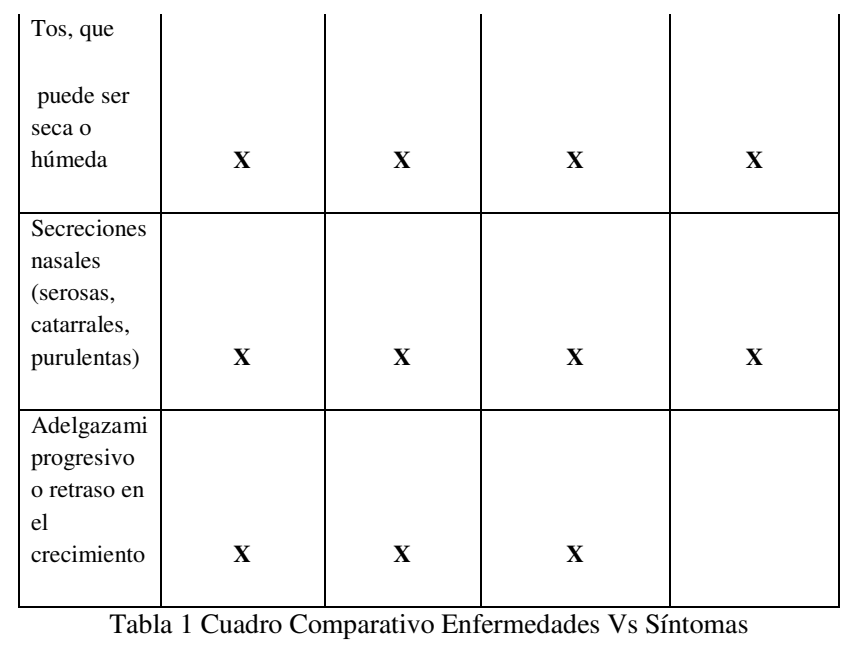

VII. CONCLUSIONES

Este sistema experto apoyara en cierto modo a la producción porcina para disminuir el tiempo en que es tratado un cerdo y que así mismo este sea atendido de manera eficiente, con el fin de no agravarse y morir o retrasarse en el crecimiento lo cual significa una pérdida monetaria grande en las fincas productoras.

\section{REFERENCIAS}

[1]. Boehringer Ingelheim. (2012). Solo Cerdos. Recuperado el 10 de Febrero de 2016, de http://www.solocerdos.es/index.php/applications/25enfermedades/66-ingelvac-myco2-enfermedad.

[2]. Arbeláez Salazar, O., Medina Aguirre, F. A., \& Chaves Osorio, J. (2011). Herramientas para el desarrollo rápido de aplicaciones web. Scientia et Technica, 254-258.

[3]. Rushton, J. (2008). The Economics of Animal Health and Production. London: Cabi.

[4]. Mares, V. (1994). Ambiente y Desarrollo: Panamá ante el Desafío Global/Hacia una Ganadería moderna y sostenible. Panamá: Poligráfica S.A.
[5]. Maes, D. (1996). Enzootic pneumonia in pigs. Veterinary Quarterly, 104-109.

[6]. Rodriguez Ferri, E., Gutierrez, C., Puente, V., Garcia , N., Garcia, N., \& Navas, J. (2002). PLEURONEUMONÍA PORCINA . Información Veterinaria, 35-44.

[7]. Uribe, C. (2006). Salmonelosis no tifoidea y su transmisión a través de alimentos de origen aviar. Colombia Médica, 151-158.

[8]. INSTITUTO NICARAGÜENSE DE TECNOLOGÍA AGROPECUARIA (INTA). (2010). Principales enfermedades de los cerdos. Nicaragua.

[9]. Jampour, M., Ashourzadeh, M., \& Yaghoobi, M. (2011). A Fuzzy Expert System to Diagnose Diseases with Neurological Signs in Domestic Animal. Information Technology: New Generations (ITNG), 1021-1024.

[10]. Bula, H., Aramendiz, H., Salas, D., Vergara, W., \& Villadiego, A. ( 2012). SISTEMA EXPERTO PARA EL DIAGNOSTICO DE PLAGAS Y ENFERMEDADES EN LOS CULTIVOS DE BERENJENA (Solanum Melongena L.) EN LA REGIÓN CARIBE DE COLOMBIA. Ingeniería e Innovación.

[11]. Huang, L., \& Liang, L. (2009). THE RESEARCH OF PHP DEVELOPMENT FRAMEWORK BASED ON MVC PATTERN. IEEE, 947-949

[12]. 3tres3 (2007). Coste económico de las principales enfermedades en grandes explotaciones porcinas en EEUU. Recuperado el 5 de Marzo de 2016, de https://www.3tres3.com/buscando/costeeconomico-de-las-principales-enfermedades-engrandes-explotacion_192 